\title{
Optimal System Design under Multi-Objective Decision making using De-Novo Concept: A New Approach
}

\author{
Sayanta Chakraborty \\ Research Scholar \\ NIT Agartala
}

\author{
Debasish Bhattacharya \\ Associate Professor \\ NIT Agartala
}

\begin{abstract}
In the realm of multi-objective optimization problem the decision maker only obtains a compromise solution or tradeoff solution. These trade-off solutions are the characteristics of sub-optimal, inefficient system configuration. But, it is preferable in all respect to arrive at a feasible solution which optimizes all the objectives at the same time. If this can be achieved then the system is said to be optimally designed. In modern era, the concept of optimal system design (if needed by extending the existed resources) is more important than to optimize a given system with fixed resources. Using De-Novo programming technique one can design an optimal system. The aim of this paper is to present a new approach of applying De-Novo programming technique for optimal design of a system. The applicability of the method has been illustrated through examples. The comparisons of the solutions obtained by the new approach and that of the existing method have been placed.
\end{abstract}

\section{General Terms}

De-Novo Programming, Optimization.

\section{Keywords}

De-Novo Programming, Multi-objective optimization, Optimum-path ratio, Optimal system design.

\section{INTRODUCTION}

Traditional linear programming technique is a good way of obtaining optimal allocation of fixed or limited resources. But the modern requirement has shifted from allocation of the fixed resources optimally in a given system to design of an optimal system extending the existed resources. De-Novo programming introduced by Zeleny [7,11] deals with this optimal designing of a system. Initially it was designed for single-criteria decision making, later it has been made applicable for multi-criteria decision making $[8,9]$. The technique is very much computation friendly and thus has become a popular multi-criteria decision making technique for the optimal designing of a system.

To get an insight of the De-Novo [6] technique let us first consider a multi-objective [3] linear decision-making problem.

$\operatorname{Max} Z=C x$

s. $t . A x \leq b$

$x \geq 0$

where $C \in R^{q \times n}$ and $C_{k}=\left(c_{k 1}, c_{k 2}, \ldots, c_{k n}\right)$ be the k-th row of $\mathrm{C}$ and $A \in R^{\mathrm{mXn}}$ are the matrices of dimensions $q \times n$ and $m \times n$ respectively, $b=\left(b_{1}, b_{2}, \ldots, b_{m}\right)^{T} \in R^{m}$ be the m-dimensional vectors of resources, $x=\left(x_{1}, x_{2}, \ldots, x_{n}\right)^{T} \in R^{m}$ be the n-dimensional vectors of decision variables. Here $z_{k}(x)=\sum_{j=1}^{n} c_{k j} x_{j}$ is the k-th objective.

Let, $z_{k}^{8}=\max z_{k}, k=1,2, \ldots q$, be the optimum value of the k-th objective function of the problem (1).Then $Z^{8}=\left(z_{1}^{*}, z_{2}^{*}, \ldots, z_{q}^{*}\right)$

, the vector of the corresponding $\mathrm{q}$ objective values, is called the ideal point of the system. Let X be the feasible region of the system (1) and $x^{8}=\left(x_{1}^{8}, \ldots \ldots \times n x_{2}^{8}\right) \in R^{n}$ is said to be the ideal solution if $C x^{8}=\left(C_{1} x^{8}, C_{2} X^{8}, \ldots \ldots, C_{q} x^{8}\right)=\left(z_{1, \ldots \ldots,}^{8} z_{q}^{8}\right)$

In general such an ideal solution may not be feasible since the objectives may be conflicting in nature too. Thus reaching to the ideal point under the given allocation of resources often becomes impossible. But the aim of the decision maker is to reach to the ideal point so that all the objectives could be optimized at the same time. Through De-Novo programming one can easily reach to the ideal point and correspondingly to the ideal solution by extending the existed resources under a given budgetary provision and thus the optimal design of the system could be accomplished.

Let, $\mathrm{B}$ be the total available budget and $p=\left(p_{1}, p_{2}, \ldots \ldots, p_{m}\right) \in R^{m}$

be the vector of unit prices of $m$ resources. Using the De-Novo programming concept for optimizing the system (1) one has to find $b \in R^{m}$, satisfying $p b \leq B$ such that the system

$\operatorname{Max} Z=C x$

s.t. $A x \leq b$

$x \geq 0$

is optimized. Thus the De-Novo Programming Problem [8] can be formulated as

$\operatorname{Max} Z=C x$

s. $t . A x \leq b$

$p b \leq B$ 


$$
x \geq 0
$$

The De-Novo concept of the extension of the feasible solution space is illustrated with the help of the figure-1.

where only two objectives (conflicting in nature) $Z_{1}$ and $Z_{2}, Z_{1}$ be the profit objective and ${ }^{Z_{2}}$ be the quality objective for an optimum production planning problem(say) has been considered. In the graph, $\mathrm{X}$ denotes the feasible solution space. It is clear that at the portion of the boundary namely $\mathrm{ABCD}$, both the objectives $Z_{1}$ and $Z_{2}$ have higher values in comparison with the other points of the boundary of $\mathrm{X}$ and thus those boundary points lying in $\mathrm{ABCD}$ part are considered as non-inferior points of the solution space. To reach to the optimal design $Z^{8}$, the existing resources should be extended and thus the budget B should be increased.

Now the system (2) is equivalent to

$\operatorname{Max} Z=C x$

s. $t . V x \leq B$

$x \geq 0$

where $V=p A=\left[\begin{array}{lllll}v_{1} & v_{2} & \ldots \ldots \ldots & \ldots & v_{n}\end{array}\right] \in R^{n}$ [4]. A meta-optimal problem [5] of (3) can be constructed as follows:

\section{min $V x$}

\section{$C x \geq Z^{8}$}

s.t.

$$
x \geq 0
$$

Solving (4) yields $x^{8}, B^{8}\left(=V x^{8}\right)$ and $b^{8}\left(=A x^{8}\right)$. The value ${ }^{B^{8}}$ represents the minimum budget to achieve $Z^{8}$ through ${ }^{x^{8}}$ under the allocation ${ }^{8}$ among the activities.

$$
\text { Since } B^{8} \geq B_{[5] \text { the optimum-path ratio for }}
$$
achieving the ideal performance $Z^{8}$ for a given budget level B is defined as $r^{8}=\frac{B}{B^{*}}$. Thus the optimal system design has been established $\quad(x, b, Z)$ as $\quad$ where
$x=r^{8} x^{8}, b=r^{8} b^{8}$ and $Z=r^{8} Z^{8}$.

In this paper a new approach for the solution of the De-Novo programming problem (2) has been presented. The method of solution has been illustrated through two examples. The solutions derived have been found to agree closely with the solutions obtained by Zeleny's method.

To accomplish our aim the paper has been divided into five sections. In section-1, the optimal designing of a system by De-Novo technique has been briefly discussed and the related literatures have been cited; in section-2, the new approach has been introduced; in section-3 the solution procedure of the proposed method has been discussed through two examples; in section-4, the comparison between the new approach and Zeleny's approach has been done and finally section- 5 contains the concluding remarks.

\section{NEW APPROACH OF SOLVING MULTI-OBJECTIVE DE-NOVO PROGRAMMING PROBLEM}

Let us recall the multi-objective De-Novo programming problem already introduced in (2):

$\operatorname{Max} Z=C x s . t \cdot A x \leq b, p b \leq B, x \geq 0$

Through the solution of (5) the optimal allocation of budget $B$ is made in such a way that the corresponding resource vector $b$

would maximize all the objectives

$Z=C x$ simultaneously. It is known that [4] the system (5) is equivalent to

$\operatorname{Max} Z=C x$

s.t. $V x \leq B$

$x \geq 0$

Let, $Z^{8}=\left(z_{1}^{8}, z_{2, \ldots x}^{8}, z_{q}^{8}\right)$ be the vector of the q-objective values corresponding to the ideal solution $x^{8}$. Zeleny[10] used the meta-optimal problem (4) for the optimal design of the system (6). Actually the problem (4) aims at a minimum budget which will help to achieve objective values at least as good as the ideal values. Obviously such budget requirement $B^{8}$ would be greater than or equal to the given budget ${ }^{B}$. In the new approach the same problem has been viewed in another perspective. It can be considered as a problem of finding the maximum budget required so that the system could reach at most to the ideal point $Z^{8}$. It will be seen that both the approaches are in fact equivalent in the sense that both of them lead to the same solution. Under this consideration let us put forward a new problem as follows:

$\max w=V x$

s.t. $C x \leq Z^{8}$

$x \geq 0$

Before proceeding further it is necessary to show that ${ }^{8}$ i.e. the ideal solution of system (1) is indeed the optimal solution of (7). This result has been placed in the form of a theorem.

\subsection{Theorem}

The single-objective LPP, $\max { }^{*}=V x$, s.t. $C x \leq Z^{8}, x \geq 0$
value at ${ }^{x^{8}}$, the ideal solution where $Z^{8}=C x^{8} V=p A$ 


\section{Proof}

Let $V x^{8}=B^{8}$ be the budget required to reach to the ideal point. Now it is clear that ${ }^{x^{8}}$ is a feasible solution of

$$
\begin{gathered}
\max w=V x \\
\text { s.t. } C x \leq Z^{8}{ }_{y} x \geq 0
\end{gathered}
$$

Let ${ }^{x^{x}}$ be any other feasible solution of the problem (7).To prove the theorem it is sufficient to show that $V x^{8} \geq V x^{y}$. If possible let, $V x^{g}>V x^{8}$.

Let us denote $A x^{y}=b^{z}$ and $B^{y}=p b^{y}$ then $p A x^{y}=p b^{b}{ }^{i} i \cdot e \cdot V x^{y}=B^{y}$. This together with our supposition $V x^{8}>V x^{8}$ yields $B^{8}<B^{9}$. But $B^{8}$ is the maximum budget which is required to be allocated so as to reach to the ideal point under the constraint $C x \leq Z^{8}$. Hence $B^{8} \geq B^{\prime}$ which contradicts the very assumption $V x^{y}>V x^{8}$, i.e. ${ }^{B^{8}<B^{y}}$. Therefore $V x^{8} \geq V x^{y}$ and hence $x^{8}$ is the optimal solution of the problem (7) and the theorem is proved.

\subsection{Remark}

In the proof of the theorem the unique character of the ideal point $Z^{8}$ and the ideal solution ${ }^{x^{8}}$ played the decisive role. The ideal solution is the only point common to all the constraints $C x \leq Z^{8}$. This is why the minimum value of the objective function at the lowest point of the upper envelope formed by the constraints is the same as the objective value at the upper point of the lower envelope and thus the problems (4) and (7) have the same optimum solution .In absence of such a common point of intersection of all the constraints the two problems namely $\min V x$, s.t. $C x \geq Z^{8}{ }_{y} x \geq 0$ and $\max V x$ s.t. $C x \leq Z^{8}, x \geq 0$; may not have a common optimal solution, e.g. the L.P.P

$\max z=5 x_{1}+7 x_{2}$

s.t. $x_{1}+x_{2} \leq 4$

$3 x_{1}+8 x_{2} \leq 24$

$10 x_{1}+7 x_{2} \leq 35$

$x_{1}, x_{2} \geq 0$

yields $\max z=24.8 ; x_{1}=1.6, x_{2}=2.4$; whereas the other problem obtained from the above L.P.P. by reversing the inequalities in the constraints and taking the objective as a minimizing one gives the solution

min $z=25.508 ; x_{1}=1.898, x_{2}=2.288$

\subsection{Note}

It can be seen that the value ${ }^{8}$ is also the minimum budget required to attain at least $Z^{8}$. To see this let us allocate a budget $B^{1}, B^{1}<B^{8}$ among the activities and $b^{1}$ be the corresponding resource vector. The optimal solution ${ }^{X^{8}}$ of the system (7) yields $B^{8}=V x^{8}$ and $b^{8}=A x^{8}$. Now the feasible region of system (2) corresponding to ${ }^{1}$ is a proper subset to that of $b^{8}$. Let, ${ }^{1}$ and $x^{8}$ be the optimal solutions of (2) corresponding to $b^{1}$ and $b^{8}$ respectively. The point $x^{8}$ is characterized by $Z^{8}=C x^{8}$ i.e. $\max z_{k}=z_{k}\left(x^{8}\right)$ $=c_{k} X^{8} \forall k=1,2, \ldots, q$

Therefore for any other point in the feasible region corresponding to $b^{8}, c_{k} x=z_{k}^{8}$ will not be satisfied for all $k$. Thus in particular for the point $x^{1}$, $z_{k}\left(x^{1}\right)<z_{k}\left(x^{8}\right)=z_{k}^{8}$ for at least one $\mathrm{k}$. So one can never reach to the ideal point $Z^{8}$ by allocating the budget $B^{1}<B^{8}$.

Therefore the optimal solution of (7) simultaneously maximizes all the objectives and the corresponding budget $\mathrm{Vx}^{*}$ is the minimum budget required to reach to the ideal point $Z^{8}$

Thus the optimal design of the multi-objective De-Novo programming problem (5) could be achieved by solving the system (7) instead of taking the meta-optimal problem (4).

Solving system (7) the values of $x^{8}, B^{8}\left(=V x^{8}\right)$ and $b^{8}\left(=A x^{8}\right)$ can be obtained. Obviously $B^{8} \geq B_{[5] \text {. Now }}$ to achieve the ideal performance $Z^{8}$ related to a given budget $B$ the optimum-path ratio can be defined as $r^{8}=\frac{B}{B^{2}}$. Since $B=\gamma^{8} B^{8}=r^{8} V x^{8}$ then $V x=V x^{8} Y^{8}$. Hence $x=x^{8} y^{8}$ is a solution satisfying the optimum-path ratio. Also since $x=x^{8} y^{8}$ then $A x=A x^{8} y^{8}$ and hence $b=b^{8} \gamma^{8}$ and similarly $Z=r^{8} Z^{8}$. Thus the optimal system can be designed as $(x, b, Z)$, where $x=\gamma^{8} x^{8}$, $\tilde{b}=\gamma^{8} b^{8}$ and $Z=\gamma^{8} Z^{8}$.

There are two additional types of budgets (other than $B$ and $B^{8}$ ) Considering a single objective (say the k-th one), the budget level required for producing the optimal $x_{j}^{k}$ with respect to the ${ }^{k t h}$ objective is $B_{j}^{k}$ which is the first type. The other is $B^{88}$. It is defined by $B^{88}=V x^{88}$ [5], where $x^{88}$ is the degenerate optimal solution of the system (5) in the case when the number of objectives is less than or 
equal to the number of variables, i.e. ${ }^{q \leq n}$. It can be shown that $B^{88} \geq B^{8} \geq B \geq B_{j}^{k}$, for $k=1,2, \ldots, q$.

Shi [5] introduced six types of optimum-path ratios:

$r_{1}=\frac{B^{*}}{B^{* v}} \quad r_{2}=\frac{B}{B^{* 4}} \quad r_{3}=\sum \lambda_{k} \frac{B_{j}^{k}}{B^{* v}} \quad r_{4}=r^{*}=\frac{B}{B^{*}}$, $r_{5}=\sum \lambda_{k} \frac{B_{j}^{*}}{B^{*}} r_{6}=\sum \lambda_{k} \frac{B_{j}^{R}}{B}$

They lead to six different policy considerations and optimal system designs. Comparative economic interpretations of all optimum-path ratios are dependent on the decision maker's value complex[9].

Through the following real life example the proposed approach of solution of multi-objective De-Novo programming problem is illustrated.

\subsection{Example}

Let us consider a mechanical shop having six machines whose present capacity portfolio is available, measured in machinehours per week for each machine. Each "hour" of machine capacity has its unit price according to the machine type. The current portfolio of available capacities is furnished through the table- 1 .

Also the demand of the capacity of the three products has been shown in the table- 2 .

Here three objectives have been chosen as profit objective, quality objective and worker satisfaction and all the objectives are considered to be equally important. The decision variables $x_{i}, i=1,2,3$ are also supplied, where $x_{i}, i=1,2,3$ respectively denote the number of units of products $1,2,3$ produced. Thus the multi-objective linear programming problem can be modeled as follows

$\max z_{1}=50 x_{1}+100 x_{2}+17.5 x_{3}$

$z_{2}=92 x_{1}+75 x_{2}+50 x_{3}$

$z_{3}=25 x_{1}+100 x_{2}+75 x_{3}$

s. t. $12 x_{1}+17 x_{2} \leq 1400$

$3 x_{1}+9 x_{2}+8 x_{3} \leq 1000$

$10 x_{1}+13 x_{2}+15 x_{3} \leq 1750$

$6 x_{1}+16 x_{3} \leq 1325$

$12 x_{2}+7 x_{3} \leq 900$

$9.5 x_{1}+9.5 x_{2}+4 x_{3} \leq 1075$

$x_{1}, x_{2}, x_{3} \geq 0$

The initial budget is given as $B=\sum p \tilde{b}=\$ 4658.75$ and the unit prices of the resources are $p_{1}=0.75, p_{2}=.60, p_{3}=0.35, p_{4}=.50, p_{5}=$ 1.15

$$
\text { and } p_{6}=0.65 \text {. }
$$

The above problem was considered by Zeleny[12]. The problem is to allocate the given budget ${ }^{B}$ among the activities to achieve an optimal design of the system. Let $b_{i}, i=1,2, \ldots, 6$ are the optimal resources corresponding to the given budget ${ }^{B x}$ The required changes in the resource components $b_{i}^{y} s$ could be effected by purchasing machines of more or of less capacities at their current prices. Then the DeNovo programming model of the problem is as follows:

$\max z_{1}=50 x_{1}+100 x_{2}+17.5 x_{3}$

$z_{2}=92 x_{1}+75 x_{2}+50 x_{3}$

$z_{3}=25 x_{1}+100 x_{2}+75 x_{3}$

s.t. $12 x_{1}+17 x_{2} \leq b_{1}$

$3 x_{1}+9 x_{2}+8 x_{3} \leq b_{2}$

$10 x_{1}+13 x_{2}+15 x_{3} \leq b_{3}$

$6 x_{1}+16 x_{3} \leq b_{4}$

$12 x_{2}+7 x_{3} \leq b_{5}$

$9.5 x_{1}+9.5 x_{2}+4 x_{3} \leq b_{6}$

$p_{1} b_{1}+p_{2} b_{2}+p_{3} b_{3}+p_{4} b_{4}+p_{5} b_{5}+p_{6} b_{6} \leq 4658.75$

$x_{1}, x_{2}, x_{3} \geq 0$

Now it is intended to solve the problem using the proposed approach and for this the given problem is to be re-casted in the form of system (7). This requires the determination of the ideal point $Z^{8}$.

The ideal point is determined by considering the objectives one by one together with the given constraints. The three LPPs so obtained are solved and the results are shown.

$$
\begin{aligned}
& Z_{1}: x_{1}=0, x_{2}=109.16813, x_{3}=0 ; Z_{1}=10916.813 \\
& Z_{2}: x_{1}=198.4558, x_{2}=0, x_{3}=0 ; Z_{2}=18257.933 \\
& Z_{3}: x_{1}=0, x_{2}=0, x_{3}=162.32578 ; Z_{3}=12174.433
\end{aligned}
$$

Thus the ideal point for the considered system is given by $Z^{8}=(10916.813,18257.933,12174.433)$. Now $V=p A=\left[\begin{array}{lll}23.475 & 42.675 & 28.7\end{array}\right]$ rewrite the problem (10) as follows:

$\max w=V x=23.475 x_{1}+42.675 x_{2}+28.7 x_{3}$ 


$$
\begin{aligned}
& 50 x_{1}+100 x_{2}+17.5 x_{3} \leq 10916.813 \\
& 92 x_{1}+75 x_{2}+50 x_{3} \leq 18257.933 \\
& 25 x_{1}+100 x_{2}+75 x_{3} \leq 12174.433
\end{aligned}
$$

$$
x_{1}, x_{2}, x_{3} \geq 0
$$

Solving system (11),one obtains

$x_{1}=131.3408, x_{2}=29.67690, x_{3}=78.97633$ i.e. $x^{8}=(131.3408,29.67690,78.97633)$-which is the ideal solution of the system (10) in view of the theorem 2.1. The corresponding budget requirement and resource vector are respectively given by

$$
B^{8}=V x^{8}=\left[\begin{array}{lll}
23.475 & 42.675 & 28.7
\end{array}\right]\left[\begin{array}{l}
131.3408 \\
29.67690 \\
78.97633
\end{array}\right]
$$

and

$$
b^{8}=A x^{8}=\left[\begin{array}{c}
1464.65 \\
910.182 \\
2030.155 \\
1444.344 \\
639.873 \\
1299.221
\end{array}\right]^{T}
$$

Now $\left(x^{8}, b^{8} Z^{8}\right)$ gives the optimal design of the system under the extended budgetary provision $B^{8}=\$ 6616.306$ . But if the system is to be designed under the given budgetary provision $B=\$ 4658.75$ then one has to make use of the optimum-path ratio $r^{8}=\frac{B}{B^{*}}$ introduced by Shi(1995) to find the corresponding optimal values of $x_{3}, b, Z$ maintaining the budget restriction as mentioned. Hence the optimal design of the product-mix problem i.e. $(x, b, Z)$ could be obtained by using the optimum-path ratio

$$
\begin{aligned}
& r^{8}=\frac{B}{B^{*}}=\frac{4658.75}{6616.306}=0.704 \text { and the relations } \\
& x=r^{8} X^{8}=(92.46,20.89,55.599) \\
& b=r^{8} b^{8}=\left[\begin{array}{c}
1464.65 \\
910.182 \\
2030.155 \\
1444.344 \\
639.873 \\
1299.221
\end{array}\right]^{T}
\end{aligned}
$$

and

$$
Z=r^{8} Z^{8}=(7685.436,12853.585,8570.801)
$$

Although by theorem 2.1 the solution obtained by the proposed method and that obtained by Zeleny's method are same, still for the validation of the proposed method a comparison of the results obtained by the two methods is shown in table- 3 .

From the corresponding values obtained by the two approaches it can be seen that the proposed method is an equivalent to that of Zeleny.

To see that the close agreement of the solutions obtained by the two methods in the above example is not a matter of chance, another numerical example has been solved by the two approaches and again an identical result has been derived.

\subsection{Numerical Example}

$$
\begin{aligned}
& Z_{2}=6 x_{1}+8 x_{2} \\
& \text { s.t. } \quad 4 x_{1} \leq 10 \\
& 2 x_{1}+6 x_{2} \leq 12 \\
& 12 x_{1}+4 x_{2} \leq 30 \\
& 3 x_{2} \leq 5.25 \\
& 4 x_{1}+4 x_{2} \leq 13 \\
& x_{1}, x_{2}, x_{3} \geq 0
\end{aligned}
$$$$
\max Z_{1}=400 x_{1}+300 x_{2}
$$

The initial budget is given as $B=\sum p b=1300$

and the unit prices of the resources are

$p_{1}=30, p_{2}=40, p_{3}=9.5 p_{4}=20 p_{5}=10$

Now the ideal point for the considered system (1) is given by $Z^{8}=(1187.5,22.25)$

$$
\text { and } V=p A=\left[\begin{array}{ll}
354 & 378
\end{array}\right] \text {. }
$$

For determining the optimal design of the system (12) the approach of Zeleny and the proposed one are respectively furnished as system (13) and (14).

$$
\begin{aligned}
& \min V x=354 x_{1}+378 x_{2} \\
& 400 x_{1}+300 x_{2} \geq 1187.5 \\
& 6 x_{1}+8 x_{2} \geq 22.25 \\
& x_{1}, x_{2} \geq 0
\end{aligned}
$$

and

$\max V x=354 x_{1}+378 x_{2}$

s.t.

$400 x_{1}+300 x_{2} \leq 1187.5$ 
$6 x_{1}+8 x_{2} \leq 22.25$

$x_{1}, x_{2} \geq 0$

The solutions of the systems (13) and (14) are found to be exactly same. The identical solution is given by $x_{1}^{8}=2.017857$ and $x_{2}^{*}=1.267857$ and the corresponding objective values are $\max Z_{1}=1187.5$ and $\max Z_{2}=22.25$

This example again validates the correctness of the proposed approach.

\section{Conclusion}

A remarkable shift from the traditional trade off solution of multi-objective optimization problem to De-Novo optimization towards optimal design of a system could be noticed in problems of economics, portfolio analysis, environmental, unemployment and inflation etc. Thus it is very much pertinent to carry forward the research on DeNovo programming and its solution procedures. In this perspective the present treatise attempts to find out a new approach of solving multi-objective De-Novo programming problem. It is believed that the solution procedure presented here could be implemented in the solution of other derivatives and extension $[1,2]$ of De-Novo programming. The advantage of the proposed approach is that it requires less number of variables (only slack variables) to be introduced in the solution procedure and thus reducing the processing time in comparison with the existing method.

Table 1. Current portfolio of available capacities

\begin{tabular}{|c|c|c|}
\hline Machine Type & Availability & Unit price(\$100 per hour) \\
& $(p)$ & 0.75 \\
\hline Milling machine & 1400 & 0.60 \\
\hline Lathe & 1000 & 0.35 \\
\hline Grinder & 1750 & 0.50 \\
\hline Jig saw & 1325 & 1.15 \\
\hline Drill press & 900 & 0.65 \\
\hline Band saw & 1075 & \\
\hline
\end{tabular}

Table 2. Demand of the capacity of the products

\begin{tabular}{|c|c|c|c|}
\hline Machine Type & Product 1 & Product 2 & Product 3 \\
\hline Milling machine & 12 & 9 & 8 \\
\hline Lathe & 3 & 13 & 15 \\
\hline Grinder & 10 & 0 & 7 \\
\hline Jig saw & 6 & 12 & 4 \\
\hline Drill press & 0 & 9.5 & 16 \\
\hline
\end{tabular}


Table 3. Comparison between the results obtained by the two methods

\begin{tabular}{|c|c|c|}
\hline & New Approach & Zeleny's Approach \\
\hline$b_{1}$ & 1031.1136 & 1031.40 \\
\hline$b_{2}$ & 640.768 & 640.936 \\
\hline$b_{3}$ & 1429.229 & 1429.578 \\
\hline$b_{4}$ & 1016.818 & 1017.026 \\
\hline$b_{5}$ & 450.47 & 450.609 \\
\hline$b_{6}$ & 914.65 & 914.88 \\
\hline$x_{1}^{s}$ & 92.46 & 92.48 \\
\hline$x_{2}^{8}$ & 20.89 & 20.90 \\
\hline$X_{3}^{8}$ & 55.599 & 55.61 \\
\hline$Z_{1}^{8}$ & 7685.436 & 7686.87 \\
\hline$Z_{2}^{B}$ & 12853.585 & 12855.89 \\
\hline$Z_{3}^{8}$ & 8570.801 & 8572.40 \\
\hline$B^{8}$ & 6616.306 & 6616.5631 \\
\hline
\end{tabular}

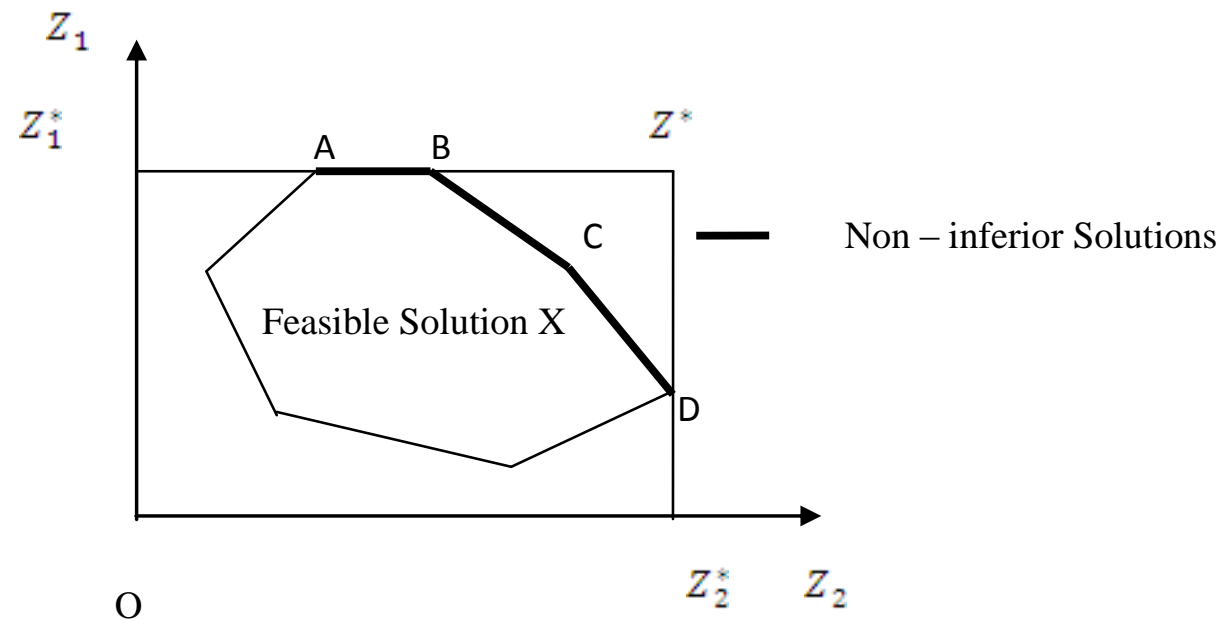

Fig 1: De - Novo programming of two objectives

\section{ACKNOWLEDGMENTS}

Our thanks to the Department of Mathematics NIT Agartala, for the contribution with resources towards the research.

\section{REFERENCES}

[1] Ankan, F., Gungor, Z., 2007, A two-phase approach for multi-objective programming problems with fuzzy coefficients, Information Sciences, (177).

[2] Certa, A., Enea, M., Galante, G., La Fata, C.M., 2009, Multi-objective human resource allocation in $\mathrm{R}$ and $\mathrm{D}$ 
projects planning, International Journal of Production Research, (47) 3503-3523.

[3] Chen, Y.W., 2004, A contractive view on multiobjective programming problems, MCDM, 6-11.

[4] Hesse,M., Zeleny, M., 1987,Optimal system designs: Towards new interpretation of shadow prices in linear programming, Computers and Operations Research,14(4),265-271.

[5] Shi, Y., 1995, Studies on optimum-path ratios in multicriteria De-Novo programming problems, Computers Math. Applic., 29(5) 43-50.

[6] Zeleny, M., 1990, De-Novo Programming, Ekonomickomatematicky obzor,(26) 406-413.

[7] Zeleny, M.,1976,Multi-objective design of highproductivity systems, In: Proc. Joint Automatic Control Conf., paper APPL9-4, New York.
[8] Zeleny, M., 1982,Multiple Criteria Decision Making. New York, McGraw-Hill.

[9] Zeleny, M., 1998, Multiple Criteria Decision Making: Eight concepts of optimality, Human Systems Management, 17(2), 97-107.

[10] Zeleny, M., 2009, On the essential multidimensionality of an Economic problem: Towards tradeoffs-free economics, Acta Universitatis Carolinae Oeconomica, (3) $154-175$.

[11] Zeleny, M., 1981, On the squandering of resources and profits via linear programming. Interfaces, 11(5), 101107.

[12] Zeleny, M., 1986, Optimal system design with multiple criteria: De-Novo Programming approach, Engineering Costa and Production Economics, (10) 89-94. 